\title{
A!
}

This is an electronic reprint of the original article.

This reprint may differ from the original in pagination and typographic detail.

Wang, Mingjun; Yan, Zheng; Song, B.; Atiquzzaman, M.

\section{AAKA-D2D: Anonymous Authentication and Key Agreement Protocol in D2D Communications}

Published in:

2019 IEEE SmartWorld, Ubiquitous Intelligence and Computing, Advanced and Trusted Computing, Scalable

Computing and Communications, Internet of People and Smart City Innovation

DOI:

10.1109/SmartWorld-UIC-ATC-SCALCOM-IOP-SCI.2019.00248

Published: 01/01/2019

Document Version

Peer reviewed version

Please cite the original version:

Wang, M., Yan, Z., Song, B., \& Atiquzzaman, M. (2019). AAKA-D2D: Anonymous Authentication and Key Agreement Protocol in D2D Communications. In 2019 IEEE SmartWorld, Ubiquitous Intelligence and Computing, Advanced and Trusted Computing, Scalable Computing and Communications, Internet of People and Smart City Innovation (pp. 1356-1362). [9060128] IEEE. https://doi.org/10.1109/SmartWorld-UIC-ATCSCALCOM-IOP-SCI.2019.00248

This material is protected by copyright and other intellectual property rights, and duplication or sale of all or part of any of the repository collections is not permitted, except that material may be duplicated by you for your research use or educational purposes in electronic or print form. You must obtain permission for any other use. Electronic or print copies may not be offered, whether for sale or otherwise to anyone who is not an authorised user. 


\title{
AAKA-D2D: Anonymous Authentication and Key Agreement Protocol in D2D Communications
}

\author{
Mingjun Wang*, Zheng Yan ${ }^{\dagger \ddagger}$, Bin Song*, Mohammed Atiquzzaman ${ }^{\S}$ \\ ${ }^{*}$ State Key Laboratory of Integrated Services Networks, Xidian University, Xi' an, Shaanxi, China \\ ${ }^{\dagger}$ State Key Lab of ISN, School of Cyber Engineering, Xidian University, Xi' an, Shaanxi, China \\ ${ }^{\ddagger}$ Department of Communications and Networking, Aalto University, Espoo, Finland

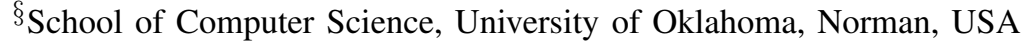 \\ Emails: \{mjwang, zyan\}@xidian.edu.cn, bsong@mail.xidian.edu.cn, atiq@ ou.edu
}

\begin{abstract}
Device-to-Device (D2D) communication is proposed as a promising technology in $5 \mathrm{G}$ system for communications between mobile devices geographical proximity. Despite significant benefits, new application scenarios and system architecture, for instance, open direct channel, expose D2D communications to unique security threats. Providing a secure and privacypreserving D2D communication system is essential for the success of D2D services. In this paper, we propose AAKA-D2D, an anonymous authentication and key agreement secure protocol for D2D communications, by which two User Equipments (UE) in close proximity could mutually authenticate without leaking their real identities and negotiate a session key for secure communications in D2D session without disclosing communication contents to Core Network (CN). Formal security analysis indicates that AAKA-D2D satisfies the security requirements. The comprehensive performance evaluation show that AAKAD2D can increase the computational performance by about $20 \%$ and decrease on communication overhead by half compared to related works.
\end{abstract}

Index Terms-Device-to-Device communication, Privacy preservation, Anonymous authentication, Key agreement

\section{INTRODUCTION}

Current demands on wireless and mobile communications motivate exploring new technology to improve network performance. Meanwhile, the appearance of new commercial services such as location-based services and content sharing services encourage us to design new paradigm to meet user demands. Device-to-Device (D2D) communication is proposed as a promising technology for communications between mobile devices geographical proximity, which is expected to play a key role in $5 \mathrm{G}$ system. D2D communication has shown great potential in improving communication capability, reducing communication delay and power dissipation, as well as fostering multifarious new applications and services. However, new application scenarios, use cases and unique system architecture of D2D communication not only provide benefits, but also lead to many security issues [1]. In conventional cellular networks, such as 3G or LTE networks [2], the communication model is centralized, which means User Equipment (UE) communicates with other UEs through the relay by Core Network (CN). The traffics are protected using Evolved Packet System Authentication and Key Agreement (EPS AKA) protocol [3] under the control of CN. Two UEs could authenticate with CN and negotiate session keys separately to protect subsequent communication sessions. However, in D2D communications, the direct D2D links between multiple UEs are novelty thus no existing scheme is available to protect the data confidentiality and integrity of D2D traffic against eavesdropping and modification by attackers. Meanwhile, authentications between UEs directly are more vulnerable to impersonation attacks. Thus, authentication and key agreement become indispensable for establishing secure D2D communications.

Many security schemes on authentication and key agreement for D2D communication have been proposed [4]-[14] recently. In these works, some schemes [4], [5] are based on security infrastructure of existing LTE networks. They adopt Key Deviation Function (KDF) and secret information stored in USIM card to achive UE authentication and derive D2D session key. However, these schemes suffer from a strong assumption that $\mathrm{CN}$ is a fully trustworthy third party. However, in many application scenarios, $\mathrm{CN}$ is curious about communication contents between UEs for commercial purposes, e.g., personalized advertisement. Other works [6][14] use heavy cryptographic methods, e.g., Diffie-Hellman Key Exchange (DHKE) [15], asymmetric encryption or digital signature algorithms, to achieve user authentication and D2D session key agreement. Among these works, [8]-[10] only address CN-absent D2D communication scenarios, which are similar to mobile adhoc network. Works [11]-[14] explore methods to secure D2D communications with joint control of CN and UE. Yan et al. [6] proposed a flexible method to address secure D2D communications with or without the support of CN. However, the utilization of cryptography makes them suffering from high computation and communication overhead.

Privacy protection is another issue in $\mathrm{D} 2 \mathrm{D}$ communications. In many D2D-based interaction scenarios, users expect their personal information, e.g., identifiers and communication content, are protected against leakage. However, most of work [4], [5], [8]-[14] don't consider this significant issue.

In order to build a security and privacy-preserving D2D communication, in this paper, we propose AAKA-D2D, a novel anonymous authentication and key agreement protocol that assures user mutual authentication, secures D2D communications and preserves user privacy simultaneously. In AAKA-D2D, a UE first subscribe to D2D communication 
system to get corresponding pseudonyms and its key pairs. Then, it requests establishing direct D2D connections with other UE under the control of CN. UE leverages Identitybased Signature (IBS) to authenticate the legality of its communication partner without learning the real identity but the pseudonym. If authentication succeeds, UEs are able to generate D2D session key by exchanging session key hints. Finally, UEs could communicate securely via direct D2D connection. In case of disputation, $\mathrm{CN}$ that acts as a session manager, can open an individual's real identity of a disputable signature. This feature encourages good behaviors of UEs and enhances security in the D2D communication system. The contributions of this paper can be summarized as follows.

- We propose AAKA-D2D, an efficient authentication and key agreement protocol for D2D communication under the joint control of $\mathrm{CN}$ and UE.

- We realize user identity anonymous by merging pseudonyms management and identity-based signature to protect user identity privacy during mutual authentication.

- We implement AAKA-D2D and verify its effectiveness and efficiency on computation and communication costs.

The rest of the paper is organized as follows. Section II reviews related work. Section III gives preliminaries, including Identity-Based Signature, Bilinear Pairing and the notations used in the paper. Section IV describes AAKA-D2D, followed by security analysis in Section $\mathrm{V}$ and performance evaluation in Section V. Finally, Section VI draws conclusions.

\section{RELATED WORK}

Recently, the security isseus in D2D communications have captured attention of many researchers. Wang et al. [1], [16] and Haus et al. [17] conducted extensive reviews on existing related work about authentication and key agreement in D2D communications.

3GPP published a Technical Specification [18] to analyze the security issues in Proximity Services (ProSe), the terminology of D2D communication in 3GPP specification. The specification provides system security requirements for different application scenarios and also includes some security mechanisms to achieve these requirements. But the security mechanisms listed are elementary and most them are desinged for a specific security reuiremetns. Comprehensive security scheme is missing.

A number of authentication and key agreement solutions have been proposed [4], [5], [8]-[14]. Alam et al. [5] proposed a key distribution scheme for D2D communications in the LTE-A system by using XOR operation to mix the keys of two pieces of D2D UEs in order to avoid the risky session key transmission between $\mathrm{CN}$ and UE. Wang et al. [4] proposed a series of key agreement and authentication protocols that support user roaming and inter-operator communications. However, both methods base on a strong security assumption that $\mathrm{CN}$ is fully trusted.

Some researches [8]-[10] focused on securing D2D communications when $\mathrm{CN}$ is absent. Sheng et al. [8] proposed a secret key establishment protocol for D2D communications based on DHKE. Goratti et al. [9] proposed a security communication protocol to establish direct links among devices by broadcasting beacon to nearby devices. Kwon et al. [10] proposed two protocols for D2D secure key establishment and authentication based on Bluetooth Pairing by using Ciphertext Policy Attribute-Based Encryption (CP-ABE) [19]. However, these methods are only appropriate for scenarios when $\mathrm{CN}$ is absent. Wang et al. [7] proposed two protocols to handle D2D group communication security. Zhang et al. [11], [13] proposed two secure data sharing and transmission protocols for D2D communications in LTE-A with joint control of $\mathrm{CN}$ and UE. However, these two protocols only support $\mathrm{CN}$-present scenarios. Moreover, Zhang's protocol was designed for data sharing, but unscalable for securing D2D communications in a generic way.

Some schemes [6], [12], [14] have been proposed to ensure secure D2D communicaitons with or without the support of $\mathrm{CN}$. Hsu et al. [12], [14] proposed two protocols to provide accountable and anonymous D2D communication establishment. However, both suffer from heavy computation and communication cost, and cannot support user privacy. Recently, Yan et al. [6] proposed a trust-based secure D2D communicaiton scheme to secure D2D communications with or without CN's appearance. Moreover, it also uses trust levels instead of real identity to protect user privcy. However, this scheme relies on an external trust management system, which pares down the applicability of this scheme.

In order to solve the privacy and computational open issues, we attempt to design an authentication and key agreement protocol for two-user D2D communications with privacy preservation in this paper.

TABLE I

DEFINICATION OF NOTATIONS

\begin{tabular}{c|l}
\hline Notations & Description \\
\hline$C N$ & The Core Network \\
$U E_{n}$ & The User Equipment $n$ \\
$R I D_{n}$ & The real identifier of $U E_{n}$ \\
$P I D_{n}$ & The pseudonym of $U E_{n}$ \\
$S I D_{i}$ & The identifier of D2D session $i$ \\
$P K_{n}$ & The public key of $U E_{n}$ associated with $P I D_{n}$ \\
$S K_{n}$ & The private key of $U E_{n}$ associated with $P I D_{n}$ \\
$K_{p u b}$ & The system public key \\
$M S K_{i}$ & The master key of D2D session $S I D_{i}$ \\
$S S K_{i}$ & The sub-session key of D2D session $S I D_{i}$ \\
$E n c(\cdot)$ & The symmetric encryption algorithm \\
$\operatorname{Dec}(\cdot)$ & The symmetric decryption algorithm \\
\hline
\end{tabular}

\section{PRELIMINARIES}

\section{A. Bilinear Pairing}

Let $G_{1}$ and $G_{2}$ be two cyclic multiplicative groups with the same prime order $q$. Discrete Logarithm Problem (DLP) is assumed to be hard in both $G_{1}$ and $G_{2}$. Let $g_{1}$ and $g_{2}$ be two generators of $G_{1}$ and $G_{2}$ respectively. Let us have a bilinear map $e: G_{1} \times G_{1} \rightarrow G_{2}$, with the following properties:

- Bilinear: For all $R, S \in G_{1}$ and $a, b \in \mathbb{Z}_{q}{ }^{*}, e\left(R^{a}, S^{b}\right)=$ $e(R, S)^{a b}$; 


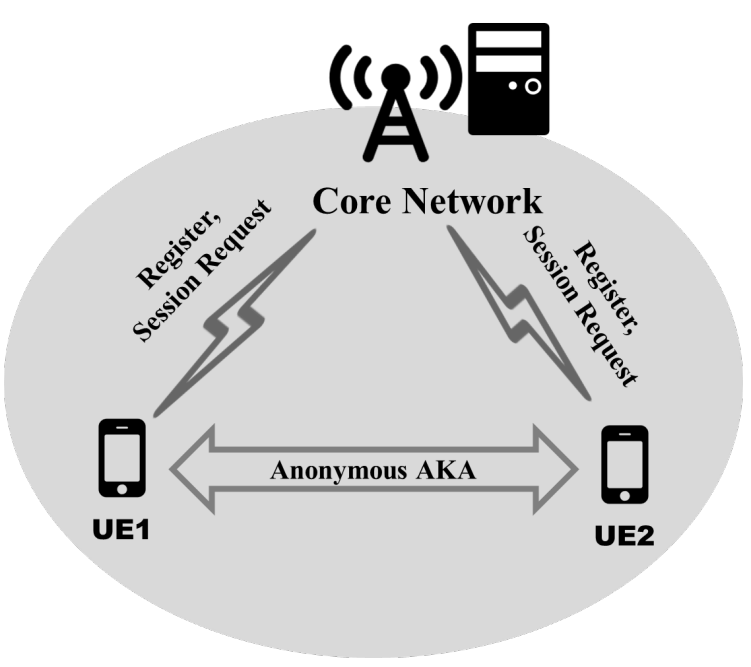

Fig. 1. System Model

- Nondegenerate: There exist $R, S \in G_{1}$ such that $e(R, S)$ $\neq 1_{G_{2}}$

- Computable: There is an efficient algorithm to compute $e(R, S)$ for any $R, S \in G_{1}$;

For example, we can construct bilinear map $e$ by the modified Weil or Tate pairings on elliptic curves [20].

\section{B. Identity-Based Signature}

Identity-Based Cryptosystem (IBC) allows the public key of an entity to be derived from its public identity information such as name, email address, etc. Shamir [21] first proposed a concept of identity-based cryptography and constructed an Identity-Based Signature (IBS) scheme using RSA function. In our system, IBS scheme is used to achieve the mutual identity authentication among UEs and also message authentication. Compared to the conventional PKI, IBS infrastructure avoids the use of certificates for public key verification and the exchange of public keys (and associated certificates). Therefore it greatly improve the efficiency of computation and communication.

For easy presentation, the notations used in this paper are listed in Table I.

\section{The Proposed Protocol}

In this section, we first describe the system model to which AAKA-D2D is applied and define security assumptions for our protocol design. Then, we present AAKA-D2D in detail, consisting of four phases: System Initialization, User Registration, D2D Discovery, Secure and Anonymous D2D Session Establishment.

\section{A. System and Security Model}

The system model is given in Fig. 1. In the system, there are two kinds of entities: 1) Core Network (CN) and 2) D2D User Equipment (UE). Herein, both UEs are located within the wireless network coverage area. Each UE has established a secure connection with $\mathrm{CN}$ via existing security solutions,

\begin{tabular}{l}
\hline \multicolumn{1}{c}{$U N E_{i}$} \\
\hline System setup phase \\
$\begin{array}{l}\text { Generate master key } s ; \\
\text { system parameters params }= \\
\left\{G_{1}, G_{2}, e, H_{1}, H_{2}, g, q, K_{p u b}, E n c_{K}(\cdot)\right\} .\end{array}$ \\
User registration phase \\
$\begin{array}{l}\text { Generate pseudonym } P I D_{i} ; \\
\text { User key pair } P K_{i} / S K_{i} .\end{array}$ \\
\hline
\end{tabular}

Fig. 2. System Setup and User Registration Phase

i.e. EPS AKA [3] in LTE network. UE can discover other UEs in the vicinity and exchange messages on insecure links, on which data confidentiality and integrity cannot be guaranteed. $\mathrm{CN}$ is assumed as a trusted party that is responsible for setting up the system, generating key pairs for UE, managing D2D communication sessions and tracing the real identity of UE. CN is supposed to have sufficient storage and powerful computation capability to perform the role of manager to support various D2D services. However, for various reasons that could offer benefits to $\mathrm{CN}$, we assume the $\mathrm{CN}$ to be curious about the contents exchanged in D2D communication sessions. More specifically, a D2D UE would like to establish a secure D2D communication with another UE in the vicinity for the purpose of D2D services, such as content sharing, and gaming. The UEs authenticate with each other and negotiate a common session key, which should only be known by D2D session users in order to protect D2D communication confidentiality against curious $\mathrm{CN}$ and attackers. Moreover, the real identities of UE are unrevealed to other UEs (includs the communication partiner UE) and attackers.

\section{B. System Initialization}

In this phase, $\mathrm{CN}$ generates system parameters. The following steps are executed by $\mathrm{CN}$ as shown in Fig. 2:

On input of security parameter $1^{n}$, CN generates a tuple $\left(G_{1}, G_{2}, e, g, q\right)$ as defined in Section Preliminaries. Then it chooses two cryptographic hash functions $H_{1}:\{0,1\}^{*} \rightarrow G_{1}$ and $H_{2}: G_{1} \times\{0,1\}^{*} \times G_{2} \rightarrow \mathbb{Z}_{q}{ }^{*}$. It picks a random number $s \in \mathbb{Z}_{q}{ }^{*}$ as system master key, computes $K_{p u b}=g^{s}$ as system public key and chooses one secure symmetric encryption algorithm $E n c(\cdot)$ (e.g., Advanced Encryption Standard (AES)). Finally, $\mathrm{CN}$ publishes the system parameters params $=\left\{G_{1}, G_{2}, e, H_{1}, H_{2}, g, q, K_{p u b}, \operatorname{Enc}(\cdot)\right\}$ and keeps $s$ a secret.

\section{User Registration}

$U E_{i}$ with real identifier $R I D_{i}$ firstly registers to $\mathrm{CN}$ for D2D service. The following steps should be performed in turn:

$U E_{i}$ sends a registration request including its real identifier $R I D_{i}$ to $\mathrm{CN}$. Once receiving the request, $\mathrm{CN}$ generates a pseudonym $P I D_{i}$ for $U E_{i}$. The pseudonym plays the same 


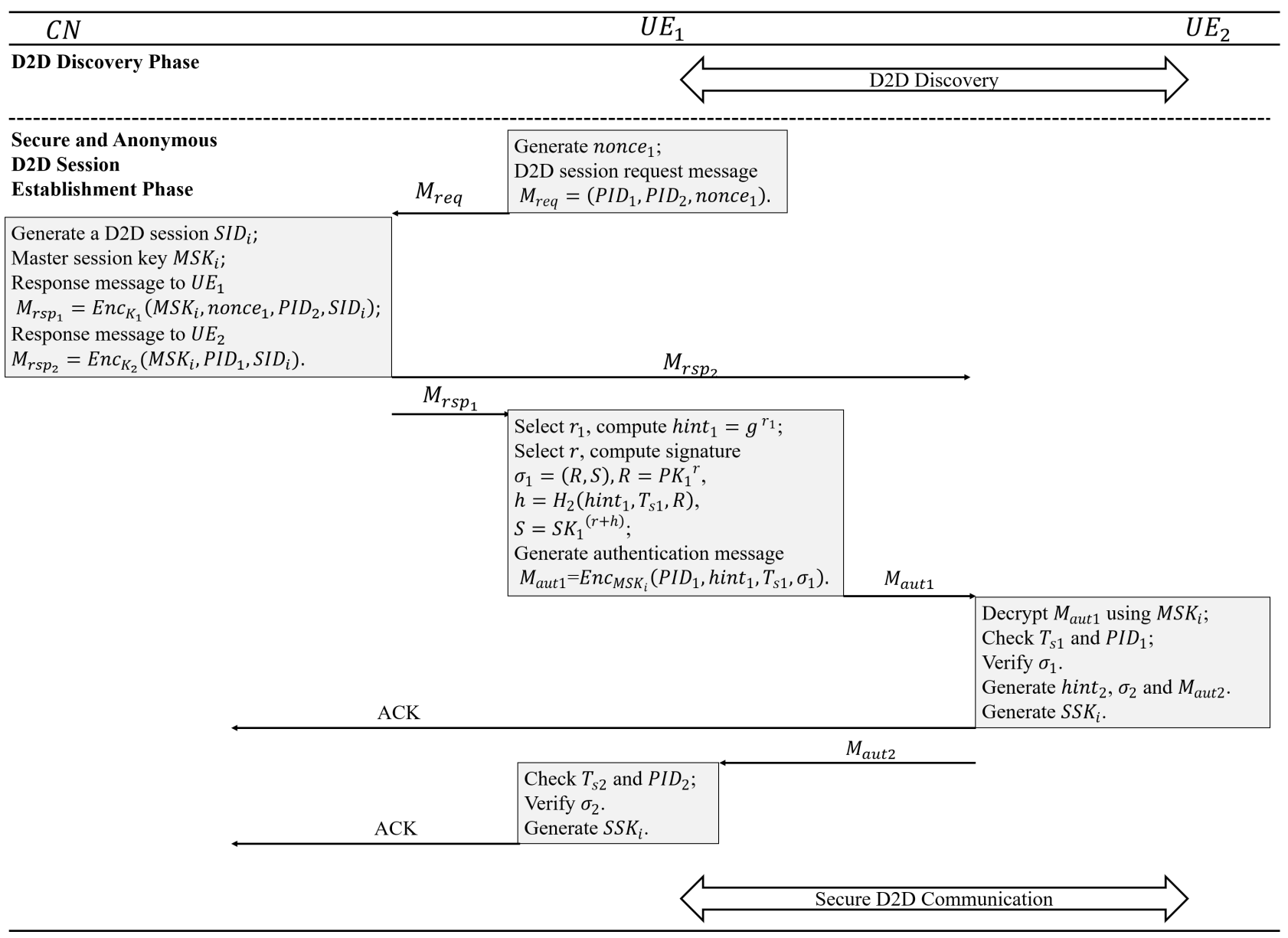

Fig. 3. Secure and Anonymous D2D Session Establishment Phase

role as real identity in authentication and secure communications with privacy-preserving support. We define the form of pseudonym as below:

$$
\text { PID } D_{i} \doteq(\text { Pseudonym, ExpiryTime })
$$

where ExpiryTime denotes the validity period of $P I D_{i} . \mathrm{CN}$ computes the public/private key pair associating with $P I D_{i}$ for $U E_{i}$, where public key $P K_{i}=H_{1}\left(P I D_{i}\right)$ and private key $S K_{i}=P K_{i}{ }^{s}$. CN send $P I D_{i}$, the corresponding key pair and the system parameters to $U E_{i}$ via the existing secure channel. In addition, $\mathrm{CN}$ locally maintains a table to manage the related information of UE, i.e., the real identity $R I D_{i}$, the pseudonyms $P I D_{i}$ and the corresponding key pair $P K_{i} / S K_{i}$, which are shown in Table II.

\section{D2D Discovery}

As shown in Fig. 3, $U E_{1}$ discovers $U E_{2}$ with pseudonym $P I D_{2}$ through a D2D discovery process. More details about the D2D discovery process can be found in [22]. After the discovery, $U E_{1}$ and $U E_{2}$ start to establish a secure D2D communication channel.

\section{E. Secure and Anonymous D2D Session Establishment}

In this phase, $U E_{1}$ and $U E_{2}$ perform anonymous mutual authentication and D2D session key agreement under the joint control of $\mathrm{CN}$ and themselve.

$U E_{1}$ generates a random number nonce $_{1}$ and sends a D2D session establishment request message $M_{r e q}$ to $\mathrm{CN}$, consisting of the pseudonyms of $U E_{1}$ and $U E_{2}$ and random number nonce $_{1}: M_{\text {req }}=\left(P I D_{1}, P I D_{2}\right.$, $\left._{\text {nonce }}\right)$.

When $\mathrm{CN}$ receives $M_{\text {req }}$, it checks ExpiryTime for the time validity of $P I D_{1}$ and $P I D_{2}$. CN rejects the request if any pseudonym is out of date. Otherwise, $\mathrm{CN}$ processes as follows:

1) Creates a new D2D session with identifier $S I D_{i}$ and adds this item into a session management table, shown in Table II.

TABLE II

D2D SESSION LIST

\begin{tabular}{c|c|c|c}
\hline $\begin{array}{c}\text { D2D } \\
\text { Session }\end{array}$ & Members & $\begin{array}{c}\text { Master } \\
\text { Session Key }\end{array}$ & $\begin{array}{c}\text { Sub-Session } \\
\text { Key }\end{array}$ \\
\hline$S I D_{i}$ & $\left(U E_{1}, U E_{2}\right)$ & $\left(M S K_{i}\right)$ & $\left(S S K_{i}\right)$ \\
\hline
\end{tabular}


2) Randomly selects a master session key $M S K_{i}=v \in \mathbb{Z}_{q}^{*}$ for $S I D_{i}$, where $M S K_{i}$ is used for secure key agreement between $U E_{1}$ and $U E_{2}$.

3) Encrypts $M S K_{i}$, nonce $_{1}$, received pseudonym of $U E_{2}$ and the session identifier $S I D_{i}$ using $\operatorname{Enc}(\cdot)$ with $K_{1}$. Herein, $K_{1}$ is a pre-load shared key between $\mathrm{CN}$ and $U E_{1}$. Then $\mathrm{CN}$ replies to $U E_{1}$ 's request by sending $M_{r s p_{1}}=E_{n C_{K_{1}}}\left(M S K_{i}\right.$, nonce $\left._{1}, P I D_{2}, S I D_{i}\right)$ and send $M_{r s p_{2}}=E_{n c_{K_{2}}}\left(M S K_{i}, P I D_{1}, S I D_{i}\right)$ to $U E_{2}$ using key $K_{2}$, where $K_{2}$ is a pre-load shared key between $\mathrm{CN}$ and $U E_{2}$.

Having received the response, $U E_{1}$ first decrypts message $M_{r s p_{1}}$ using $K_{1}$. Then, it randomly selects $r_{1} \in \mathbb{Z}_{q}^{*}$ and computes hint $_{1}=g^{r_{1}}$ as as its key hint. Moreover, it picks a random number $r \in \mathbb{Z}_{q}^{*}$ and computes a signature $\sigma_{1}$ using its key pair $P K_{1} / S K_{1}$ as follows:

$$
\sigma_{1}=(R, S),
$$

where $R=P K_{1}^{r}, h=H_{2}\left(\right.$ hint $\left._{1}, T_{s 1}, R\right)$ and $S=S K_{1}{ }^{r+h}$, $T_{s 1}$ is timestamp. After computing the signature, $U E_{1}$ encrypts tuple $\left(P I D_{1}\right.$, hint $\left._{1}, T_{s 1}, \sigma_{1}\right)$ using D2D session master key $M S K_{i}$ and sends authentication message $M_{\text {aut } 1}=$ $\operatorname{Enc}_{M S K_{i}}\left(P I D_{1}\right.$, hint $\left._{1}, T_{s 1}, \sigma_{1}\right)$ to $U E_{2}$.

Upon receiving the authentication message $\overline{M_{a u t 1}}$ from $U E_{1}, U E_{2}$ decrypts the message using $M S K_{i}$. Then it checks whether the timestamp $\overline{T_{s 1}}$ is valid and the pseudonym $\overline{P I D_{1}}$ is the same as the one received from $\mathrm{CN}$. If both checks pass, $U E_{2}$ verifies the signature as below:

1) Compute $\overline{P K_{1}}=H_{1}\left(\overline{P I D_{1}}\right) ; \bar{h}=H_{2}\left(\overline{\text { hint }_{1}}, \overline{T_{s 1}}, \bar{R}\right)$.

2) Verify $e(g, \bar{S}) \stackrel{?}{=} e\left(K_{p u b},\left(\overline{P K_{1}}\right)^{\bar{h}} \cdot R\right)$.

Below we show why verification succeeds if all parameters are created as explained:

$$
\begin{aligned}
\text { LHS } & =e(g, S) & R H S & =e\left(K_{\text {pub }}, P K_{1}{ }^{h} \cdot \bar{R}\right) \\
& =e\left(g, S K_{1}{ }^{(r+h)}\right) & & =e\left(g^{s}, P K_{1}{ }^{h} \cdot P{K_{1}}^{r}\right) \\
& =e\left(g, P K_{1}{ }^{(r+h) s}\right) & & =e\left(g^{s}, P K_{1}{ }^{(h+r)}\right) \\
& =e\left(g, P K_{1}\right)^{(r+h) s} & & =e\left(g, P K_{1}\right)^{(h+r) s}
\end{aligned}
$$

If the verification succeeds, $U E_{2}$ generates authentication message $M_{\text {aut } 2}$, similarly as what $U E_{1}$ does and sends it to $U E_{1}$ for authentication. Furthermore, $U E_{2}$ sends $\mathrm{CN} A C K$ message.

Upon receiving $U E_{2}$ 's authentication message, $U E_{1}$ verifies the timestamp, the pseudonym and the signature. If all verifications pass, $U E_{1}$ sends $A C K$ message to $C N$. Meanwhile, both $U E_{1}$ and $U E_{2}$ get a sub-session key for D2D communications by computing $S S K_{i}=\left(g^{r_{1}}\right)^{r_{2}}=\left(g^{r_{2}}\right)^{r_{1}}=g^{r_{1} r_{2}}$. With $S S K_{i}, U E_{1}$ and $U E_{2}$ could communication securely via D2D link.

\section{Security Analysis}

In this section, we theoretically analyze the security of AAKA-D2D against three security objectives: mutual authentication, secure session key generation, and privacy preservation.

\section{A. Mutual Authentication}

In the proposed protocol, in order to guarantee the validity of identities of the communication parties, mutual authentications should be performed between $\mathrm{UE}$ and $\mathrm{CN}$, and also between two UEs. Since our protocol inherits the security architecture of EPS by introducing security features for D2D communications into it, the mutual authentication between UE and $\mathrm{CN}$ can be achieved based on the EPS AKA protocol [3]. We adopt identity-based signature to achieve mutual authentication between UEs. Each UE computes its signature $\sigma_{1}=(R, S)$ for time-stamped key hint by using its public key, private key and the random $r$. Attacker has no knowledge of the private key and the random number chosen by UE, and thus cannot forge signature. The time-stamp prevents from simply replaying a recorded valid signature. Only the legitimate UE with the correct private key and the secret random number can compute a valid signature.

\section{B. Secure Session Key Generation (Secrecy of Session Key)}

To protect the communications over the direct D2D link between two UEs, a distinct session key should be negotiated for each D2D session with the cooperation of two UEs and $\mathrm{CN}$. The session key is generated by carrying out the DHKE algorithm between two pieces of UE. $U E_{1}$ and $U E_{2}$ use their random secret $r_{1}$ and $r_{2}$ to generate their session key hints; then to generate the session key. Thus, only legitimate UE keeping secrets can generate the session key. Moreover, CN provides master session key $M S K_{i}$ for each UE to secure communications between UEs at the session key generation phase. The UE that has no knowledge of $M S K_{i}$ cannot get the session key generation materials in messages $M_{a u t 1}$ and $M_{\text {aut } 2}$. On the other hand, $\mathrm{CN}$ cannot get the random secrets of UE to generate the session key because of the intractability of the computational Diffie- Hellman problem. Thus, AAKAD2D preserves the D2D communication privacy from honestbut-curious $\mathrm{CN}$ and malicious attackers. At last, a session ID is used in the session key generation in order to guarantee the uniqueness of the session key for a specific D2D session.

\section{Privacy Preservation}

For AAKA-D2D, we analyze two aspects of the privacy preservation: communication content privacy and identity privacy. For communication contents, in the current security solutions in LTE, communications between two UEs must be routed through the core network, therefore, communication contents between UE can be protected against other UEs but they are open to $\mathrm{CN}$. It means the privacy of communication contents between UE are under risk if the core network is compromised. But in AAKA-D2D, both the attackers and CN are unable to get the plaintext, even though $\mathrm{CN}$ participates in key generation process. For identity privacy, pseudonyms are used instead of real identities in the D2D discovery phase, the session establishment phase and, later on, in the D2D session communication. Only $\mathrm{CN}$ is able to retrieve the real identity from a pseudonym of a UE. UE has no ability to get the real identity from the pseudonym. For different D2D sessions, UE 
TABLE III

COMPUTATION OVERHEAD COMPARISONS

\begin{tabular}{|c|c|c|c|c|}
\hline & Steps & AAKA-D2D & SeDS [11] & CN-GD2C [14] \\
\hline \multirow{3}{*}{$C N$} & System Setup & $1 * \operatorname{Rand}+1 * \operatorname{Exp}$ & $1 *$ Rand $+1 * \operatorname{Exp}$ & $1 *$ Hash \\
\hline & User Regist & $1 * E x p+1 *$ Hash & $1 *$ Rand $+1 * \operatorname{Exp}+1 *$ Hash & $1 * E x p+1 *$ Hash \\
\hline & $\begin{array}{l}\text { Session } \\
\text { Establish }\end{array}$ & $1 * \operatorname{Rand}+1 * \operatorname{Enc}(\cdot)$ & $1 * \operatorname{Rand}+2 * \operatorname{Exp}+1 * \operatorname{Dec}(\cdot)$ & $\begin{array}{l}6 * \operatorname{Hash}+2 * \operatorname{Pair}+ \\
2 * \operatorname{Enc}(\cdot)+2 * \operatorname{Dec}(\cdot)\end{array}$ \\
\hline \multirow[b]{2}{*}{$U E$} & User Regist & - & - & - \\
\hline & $\begin{array}{l}\text { Session } \\
\text { Establish }\end{array}$ & $\begin{array}{l}2 * \text { Rand }+5 * \operatorname{Exp}+3 * \text { Hash }+ \\
1 * \operatorname{Enc}(\cdot)+2 * \operatorname{Dec}(\cdot)+2 * \text { Pair }\end{array}$ & $\begin{array}{l}2 * \operatorname{Rand}+5 * \operatorname{Exp}+4 * \text { Hash }+ \\
1 * \operatorname{Enc}(\cdot)+2 * \operatorname{Dec}(\cdot)+4 * \text { Pair }\end{array}$ & $\begin{array}{c}2 * \text { Rand }+8 * \operatorname{Exp}+5 * \text { Hash } \\
\quad+2 * \operatorname{Enc}(\cdot)+3 * \operatorname{Dec}(\cdot)\end{array}$ \\
\hline
\end{tabular}

could request different pseudonyms from $\mathrm{CN}$ and use them for privacy preservation. The link between different pseudonyms of one UE can only be revealed by $\mathrm{CN}$.

\section{Performance Evaluation}

In this section, we first analyze the performance of AAKAD2D in terms of computation complexity and communication cost. Then, we evaluate the performance based on protocol implementation. We also compare the performance of AAKAD2D with two existing authentication and key agreement D2D communication protocols [11], [14] to show the efficiency of our protocol.

\section{A. Efficiency Analysis}

We analyze the efficiency of the protocol in terms of the computation complexity and communication overhead.

1) Computation complexity: AAKA-D2D involves two types of system entities, i.e., CN and UE. We analyze the computation cost of each type and compare the computation complexity with SeDS [11] and CN-GD2C [14]. In [11], the Service Provider (SP) and the evolved NodeB (eNB) play the same role as $\mathrm{CN}$ and in [14], eNB, ProSe Function and $\mathrm{HSS} / \mathrm{AuC}$ play the same role as $\mathrm{CN}$ in our protocol. Thus, we analyze SeDS by combining SP and eNB together and $\mathrm{CN}-\mathrm{GD} 2 \mathrm{C}$ by combining eNB, ProSe Function and HSS/AuC together.

Table III shows the computation complexity analysis of AAKA-D2D based on each step and the comparison with $\mathrm{SeDS}$ and CN-GD2C. We can see that the computation complexity of AAKA-D2D is lower than SeDS on all operations both at CN and UE. Compared with CN-GD2C, AAKA-D2D has lighter computation complexity on all operations at $\mathrm{CN}$ but has two more paring operations at UE.

2) Communication overhead: We analyze the communication overhead in the system from two aspects: data transmitted between $\mathrm{CN}$ and UE and that between UEs. In AAKA-D2D, the communication overhead between $\mathrm{CN}$ and UE in user registration phase only occurs once when user joins the system, so we do not take into account this overhead. In SeDS and CNGD2C, we also treat related entities in core network as $\mathrm{CN}$ as we does above. Table IV shows the comparison of communication overhead. We can find from the table that the communication overhead between $\mathrm{UE}$ and $\mathrm{CN}$ in our
TABLE IV

COMMUNICATION OVERHEAD COMPARISONS

\begin{tabular}{c|c|c|c}
\hline $\begin{array}{c}\text { Communication } \\
\text { Overhead (Bytes) }\end{array}$ & $\begin{array}{c}\text { AAKA- } \\
\text { D2D }\end{array}$ & $\begin{array}{c}\text { SeDS } \\
{[8]}\end{array}$ & $\begin{array}{c}\text { CN-GD2C } \\
{[\mathbf{1 1}]}\end{array}$ \\
\hline $\begin{array}{c}\text { Between UE } \\
\text { and CN (eNB) }\end{array}$ & 33 & 302 & 250 \\
\hline Between UEs & 126 & 48 & 86 \\
\hline Total & 159 & 350 & 336 \\
\hline
\end{tabular}

protocol is less than about 200 Bytes compared with SeDS and CN-GD2C, but the communication overhead between UEs is heavier than the others two protocols. However, the total communication overhead of our protocol is only half of SeDS and $\mathrm{CN}-\mathrm{GD} 2 \mathrm{C}$, which reduces about 150 Bytes.

\section{B. Simulation and Evalution}

In this subsection, we illustrate the performance testing result of AAKA-D2D based on simulation. Herein, we applied pairing-based cryptography library (PBC) [23] for algebraicoperations and OpenSSL [24] for cryptography operations and communication transmission. The simulation used a 160bit elliptic curve group based on the super-singular curve $y^{2}=x^{3}+x$ over a base 512-bit finite field. On our testing machine, the pairing operation can be computed in approximately 3.8 milliseconds (ms) (without preprocessing), and the exponentiation computations in $G_{1}$ and $G_{2}$ take about $3.4 \mathrm{~ms}$ and $0.5 \mathrm{~ms}$, respectively. Random element selection is also a significant operation, which takes about $4.5 \mathrm{~ms}$ for $G_{1}$ and $2.2 \mathrm{~ms}$ for $G_{2}$.

Table $\mathrm{V}$ shows the operation time comparisons. As we can see from the table, the most time-consuming operation for $\mathrm{UE}$ is the session key generation. It involves some heavy operations, i.e., pairing and exponentiation operations. Compared with SeDS and CN-GD2C, the computation overheads of $\mathrm{UE}$ and $\mathrm{CN}$ in our protocol are lighter in general than these in SeDS and CN-GD2C. In our protocol, CN works in System Setup and User Registration steps rather than all three processing steps as happens in SeDS and CN-GD2C, which decreases computation overhead of $\mathrm{CN}$. We can also see that the total performance of computation cost of AAKA-D2D are better with 23\% decrease than SeDS and 19\% than CN-GD2C. 
TABLE V

OPERATION TIME COMPARISONS

\begin{tabular}{c|c|c|c|c|c}
\hline \multirow{2}{*}{} & \multirow{2}{*}{ Entity } & \multicolumn{4}{|c}{ Operation Time (ms) } \\
\cline { 3 - 6 } & & $\begin{array}{c}\text { System } \\
\text { Setup }\end{array}$ & $\begin{array}{c}\text { User } \\
\text { Regist }\end{array}$ & $\begin{array}{c}\text { Session } \\
\text { Key Gene }\end{array}$ & Total \\
\hline \multirow{2}{*}{$\begin{array}{c}\text { AAKA- } \\
\text { D2D }\end{array}$} & $C N$ & 12.1 & 6.0 & - & \\
& $U E_{1}$ & - & - & 29.0 & 75.9 \\
& $U E_{2}$ & - & - & 28.8 & \\
\hline \multirow{2}{*}{ SeDS } & $C N$ & 16.6 & 10.5 & 11.5 & \\
{$[11]$} & $U E_{1}$ & - & - & 31.3 & 98.3 \\
& $U E_{2}$ & - & - & 28.4 & \\
\hline \multirow{2}{*}{ CN-GD2C } & $C N$ & 12.3 & 6.0 & 7.8 & \\
{$[\mathbf{1 4}]$} & $U E_{1}$ & - & - & 34.5 & 93.3 \\
& $U E_{2}$ & - & - & 32.7 & \\
\hline
\end{tabular}

\section{CONCLUSION}

In this paper, we propose AAKA-D2D, a novel anonymous authentication and key agreement protocol that achieves user anonymous authentication, secures D2D communications, and preserves user privacy simultaneously. Two UEs subscribing to D2D communication service could discover each other in close proximity, establish a secure D2D direct communication link by adopting the proposed protocol. They use pseudonyms and secret key pairs obtained from $\mathrm{CN}$ to perform privacypreserving mutual authentication and take advantage of DHKE to negotiate a session key for subsequent D2D direct communications. Analysis on security proves AAKA-D2D meets the security requirments. Performance analysis and experimental results show that the proposed protocol is efficient with regard to both computation and communication.

\section{ACKNOWLEDGMENT}

This work is sponsored by the NSFC (grants 61672410, 61802293 and U1536202), Academy of Finland (grants 308087 and 314203), the National Key Research and Development Program of China (grant 2016YFB0800704), National Postdoctoral Program for Innovative Talents (grant BX20180238), the Project funded by China Postdoctoral Science Foundation (grant 2018M633461), the Key Lab of Information Network Security, Ministry of Public Security under grant No.C18614, the Fundamental Research Funds for the Central Universities (grant JB191504), and the 111 project (grant B16037).

\section{REFERENCES}

[1] M. Wang and Z. Yan, "A survey on security in D2D communications," Mobile Networks and Applications, vol. 22, pp. 195-208, Apr. 2017.

[2] S. Stefania, T. Issam, and B. Matthew, "LTE, the UMTS long term evolution: from theory to practice," A John Wiley and Sons, Ltd, vol. 6, pp. 136-144, 2009.
[3] 3GPP, "3GPP system architecture evolution(SAE) (Rel 15)," Technical Standard(TS) 33.401, 3rd Generation Partnership Project, Jun. 2018. V15.5.0.

[4] M. Wang, Z. Yan, and V. Niemi, "UAKA-D2D: Universal authentication and key agreement protocol in D2D communications," Mobile Networks and Applications, vol. 22, pp. 510-525, Jun 2017.

[5] M. Alam, D. Yang, J. Rodriguez, and R. A. Abd-alhameed, "Secure Device-to-Device communication in LTE-A," IEEE Communications Magazine, vol. 52, pp. 66-73, April 2014.

[6] Z. Yan, H. Xie, P. Zhang, and B. B. Gupta, "Flexible data access control in D2D communications," Future Generation Computer Systems, vol. 82 , pp. $738-751,2018$.

[7] W. Mingjun and Y. Zheng, "Privacy-preserving authentication and key agreement protocols for D2D group communications," IEEE Transactions on Industrial Informatics, vol. 14, pp. 3637-3647, Aug 2018.

[8] W. Shen, W. Hong, X. Cao, B. Yin, D. M. Shila, and Y. Cheng, "Secure key establishment for Device-to-Device communications," in 2014 IEEE Global Communications Conference, pp. 336-340, Dec 2014.

[9] L. Goratti, G. Steri, K. M. Gomez, and G. Baldini, "Connectivity and security in a D2D communication protocol for public safety applications," in 2014 11th International Symposium on Wireless Communications Systems (ISWCS), pp. 548-552, Aug 2014.

[10] H. Kwon, C. Hahn, D. Kim, K. Kang, and J. Hur, "Secure Deviceto-Device authentication in mobile multi-hop networks," in Wireless Algorithms, Systems, and Applications, (Cham), pp. 267-278, Springer International Publishing, 2014.

[11] A. Zhang, J. Chen, R. Q. Hu, and Y. Qian, "SeDS: Secure data sharing strategy for D2D communication in LTE-Advanced networks," IEEE Transactions on Vehicular Technology, vol. 65, pp. 2659-2672, April 2016.

[12] R. Hsu and J. Lee, "Group anonymous D2D communication with end-toend security in LTE-A," in 2015 IEEE Conference on Communications and Network Security (CNS), pp. 451-459, Sep. 2015.

[13] A. Zhang, L. Wang, X. Ye, and X. Lin, "Light-weight and robust security-aware D2D-assist data transmission protocol for mobile-health systems," IEEE Transactions on Information Forensics and Security, vol. 12, pp. 662-675, March 2017.

[14] R. Hsu, J. Lee, T. Q. S. Quek, and J. Chen, "GRAAD: Group anonymous and accountable D2D communication in mobile networks," IEEE Transactions on Information Forensics and Security, vol. 13, pp. 449464, Feb 2018.

[15] W. Diffie and M. Hellman, "New directions in cryptography," IEEE Transactions on Information Theory, vol. 22, pp. 644-654, November 1976.

[16] M. Wang and Z. Yan, "Security in d2d communications: A review," in 2015 IEEE Trustcom/BigDataSE/ISPA, vol. 1, pp. 1199-1204, Aug 2015.

[17] M. Haus, M. Waqas, A. Y. Ding, Y. Li, S. Tarkoma, and J. Ott, "Security and privacy in device-to-device (d2d) communication: A review," IEEE Communications Surveys Tutorials, vol. 19, pp. 10541079, Secondquarter 2017.

[18] 3GPP, "Proximity-based services (ProSe); Security aspects (Rel 15)," Technical Standard(TS) 33.303, 3rd Generation Partnership Project , Jun. 2018. V15.0.0.

[19] J. Bethencourt, A. Sahai, and B. Waters, "Ciphertext-policy attributebased encryption," in 2007 IEEE Symposium on Security and Privacy (SP '07), pp. 321-334, May 2007.

[20] D. Boneh and M. Franklin, "Identity-based encryption from the weil pairing," in Advances in Cryptology - CRYPTO 2001, (Berlin, Heidelberg), pp. 213-229, Springer Berlin Heidelberg, 2001.

[21] A. Shamir, "Identity-based cryptosystems and signature schemes," in Advances in Cryptology (G. R. Blakley and D. Chaum, eds.), (Berlin, Heidelberg), pp. 47-53, Springer Berlin Heidelberg, 1985.

[22] 3GPP, "Proximity-based services (ProSe); Stage 2(Rel 15)," Technical Standard(TS) 23.303, 3rd Generation Partnership Project, Jun. 2018. V15.1.0.

[23] JPBC, "The Java Pairing-Based Cryptography Library (JPBC)."

[24] OpenSSL, "Cryptography and SSL/TLS toolkit." 